\title{
Knockdown of LncRNA H19 Relieves LPS-Induced Damage by Modulating miR-130a in Osteoarthritis
}

\author{
$\mathrm{Yi} \mathrm{Hu}{ }^{1,2 *}$, Sukai $\mathrm{Li}^{3 *}$, and Yonggen $\mathrm{Zou}^{1}$ \\ ${ }^{1}$ Department of Orthopedics, The Affiliated Traditional Chinese Medicine Hospital of Southwest Medical University, Luzhou; \\ ${ }^{2}$ Department of Orthopedics, Chongqing Dongnan Hospital, Chongqing; \\ ${ }^{3}$ Department One of Orthopedics, Dianjiang People's Hospital of Chongqing, Dianjiang County, Chongqing, China.
}

Purpose: Osteoarthritis (OA) is a commonly occurring illness without a definitive cure, at present. Long non-coding RNAs (lncRNAs) have been widely confirmed to be involved in the modulation of OA progression. This study aimed to investigate the role and mechanism of IncRNA H19 in OA.

Materials and Methods: Abundances of H19 and microRNA-130a (miR-130a) in lipopolysaccharide (LPS)-treated C28/I2 cells were measured by reverse-transcription quantitative PCR (RT-qPCR). CCK-8 and flow cytometry analyses were carried out to assess cell viability and apoptosis. Starbase online software was used to predict the putative binding sites between H19 and miR-130a. Luciferase reporter, RNA pull down, and RT-qPCR were performed to analyze the true interaction between H19 and miR-130a.

Results: A notably dose-dependent elevation of H19 levels was observed in LPS-treated C28/I2 cells. Knockdown of H19 ameliorated the injury of LPS-induced C28/I2 cells, reflected by induced viability, decreased apoptosis, and reduced inflammatory factor secretions. Moreover, H19 negatively regulated the expression of miR-130a via acting as a molecular sponge for miR-130a. The stimulatory effects of $\mathrm{H} 19$ on cell damage were abolished following the restoration of miR-130a.

Conclusion: LncRNA H19 aggravated the injury of LPS-induced C28/I2 cells by sponging miR-130a, hinting a novel regulatory mechanism and a potential therapeutic target for $\mathrm{OA}$.

Key Words: Osteoarthritis, lipopolysaccharide, IncRNA H19, miR-130a

\section{INTRODUCTION}

Osteoarthritis (OA) is characterized by joint inflammation and articular cartilage destruction. ${ }^{1}$ Genetic, biochemical, and biomechanical factors are associated with the occurrence and development of $\mathrm{OA}^{2}$ As widely believed, $\mathrm{OA}$ is a leading cause for severe physical disability with pain, stiffness, swell-

Received: August 3, 2018 Revised: December 21, 2018

Accepted: December 29, 2018

Corresponding author: Yonggen Zou, MD, Department of Orthopedics, The Affiliated Traditional Chinese Medicine Hospital of Southwest Medical University, No.182, Chunhui Road, Longmatan District, Luzhou, 646000, Sichuan Province, China. Tel: 86-0830-3161916, E-mail: w0309211@sina.com

*Yi Hu and Sukai Li contributed equally to this work.

-The authors have no potential conflicts of interest to disclose.

(C) Copyright: Yonsei University College of Medicine 2019

This is an Open Access article distributed under the terms of the Creative Commons Attribution Non-Commercial License (https://creativecommons.org/licenses/ by-nc/4.0) which permits unrestricted non-commercial use, distribution, and reproduction in any medium, provided the original work is properly cited. ing, joint deformity, and dyskinesia.-5 The risk of OA appears to be related with increased age, as $63 \%$ of OA cases are 60 years of age or older. ${ }^{6}$ Meanwhile, modern therapeutic strategies for OA patients are focused on pain relief and symptom control rather than curing the disease. In view of the limited outcomes of current treatments for $\mathrm{OA}$, novel and effective strategies for $\mathrm{OA}$ are urgently needed.

Chondrocytes are the sole cell type in cartilage associated with the architecture and function of cartilage. Abnormal alteration of chondrocyte apoptosis and inflammatory response are likely responsible for cartilage degradation in OA. ${ }^{7-10}$ Lipopolysaccharide (LPS) is considered to be a pivotal pro-inflammatory factor associated with the pathogenesis of OA. ${ }^{11}$ Reliable evidence has indicated that LPS-exposure can induce inflammatory injury of chondrocytes in vitro. ${ }^{12}$ Therefore, developing a novel targeted drug to inhibit LPS-induced chondrocytes injury may help to improve OA.

Long non-coding RNAs (LncRNAs), a series of highly conserved transcripts of sizes larger than 200 nucleotides (nt) in 
length, have been shown to be involved in the modulation of pathological development in human diseases, including OA. ${ }^{13,14}$ Liu, et al. ${ }^{15}$ reported that a total of 107 IncRNAs were differentially expressed in damaged cartilage versus intact cartilage. Of these IncRNAs, 51 were upregulated and 56 were downregulated in damaged cartilage, compared to normal cartilage, indicating the underlying role of lncRNAs in OA. H19, one of the early identified maternally expressed imprinted lncRNA, is specifically located on human chromosome 11p15.5. Growing evidence has demonstrated that H19 is a vital regulator of inflammatory response and that ectopic expression of $\mathrm{H} 19$ is observed in a range of inflammation-linked diseases. A previous study reported that knockdown of H19 attenuates the inflammation response in oxidized low density lipoprotein (ox-LDL)treated Raw264.7 cells by sponging miR-130b. ${ }^{16}$ Moreover, H19 transcript is mediated in the course of a hypoxic and inflammatory challenge of cultured chondrocytes. ${ }^{17}$ However, the function and precise mechanism of $\mathrm{H} 19$ in the pathogenesis of $\mathrm{OA}$ is still far from being fully addressed.

MicroRNAs (miRNAs), similar to lncRNA, are another type of non-protein coding RNA molecule larger than 18-22 nt that are closely associated with the regulation of cell phenotypes, including cell proliferation, differentiation, and apoptosis. Recent studies have confirmed the vital roles of miR-130b in regulating inflammatory responses. ${ }^{18}$ Moreover, downregulation of miR-130a in chondrocytes has been found to induce inflammatory cytokines secretion. ${ }^{19}$ Recently, a novel mechanism suggested that IncRNAs can function as a molecular sponge for miRNAs. Here, we aimed to investigate whether H19 could regulate LPS-triggered chondrocyte growth and inflammation response by sponging miR-130a.

\section{MATERIALS AND METHODS}

\section{Cell culture and treatment}

The study was approved by the Institutional Review Board of The Affiliated Traditional Chinese Medicine Hospital of Sounthwest Medical University. The normal human cartilage cells C28/I2 and human renal epithelial cells 293T were purchased from American Type Culture Collection (ATCC, Manassas, VA, USA). 293T cells were grown in Dulbecco's Modified Eagle Medium (DMEM) (Gibco, Carlsbad, CA, USA) supplemented with $1 \%$ penicillin/streptomycin and $10 \%$ fetal bovine serum (FBS, Gibco). C28/I2 cells were cultured in DMEM-F12 medium (Gibco) with $10 \%$ FBS. All cells were maintained in a moist incubator with $5 \% \mathrm{CO}_{2}$ at $37^{\circ} \mathrm{C}$. The medium was changed every $24 \mathrm{~h}$ until cell confluence achieved $85 \%$. For LPS treatment, C28/I2 cells were exposed to 1,5 , or $10 \mu \mathrm{g} / \mathrm{mL}$ of LPS (Sigma-Aldrich, St. Louis, MO, USA) for $12 \mathrm{~h}$, with non-treated cells as a control group.

\section{Cell transfection}

Three small interfering RNAs (siRNAs) targeting H19 and scramble siRNA (si-NC) were purchased from GenePharma Co. Ltd. (Shanghai, China). The sequences were 5'-CCAACAU CAAAGACACCAUdTdT-3' for H19-siRNA1, 5'-GCAGGA CAUGACAUGGUCCdTdT-3' for H19-siRNA2, and 5'-UAAGU CAUUUGCACUGGUUdTdT-3' for H19-siRNA3. The si-H19 used in this study comprised mixtures of three siRNAs. The sequences for si-NC were 5'-UUCUCCGAACGUGUCACGUTT-3'. miR-130a mimics (miR-130a), miR-130a inhibitor (anti-miR130a), H19-overexpression plasmid pcDNA-H19 (H19), and corresponding negative controls were all purchased from $\mathrm{Ge}-$ nePharma Co. Ltd. for loss- and gain-of-function analyses.

LPS-treated C28/I2 cells were seeded into six-well plates for $24 \mathrm{~h}$ of incubation. After that, we transfected oligonucleotides or plasmids into cells using Lipofectamine 2000 (Invitrogen, Carlsbad, CA, USA) following the manufacturer's procedure. About 48-h post-transfection, cells were collected for following experiments.

\section{RNA extraction and reverse-transcription quantitative PCR}

To investigate the role of lncRNA H19 and miR-130a in LPSdamaged C28/I2 cells, the expression levels of H19 and miR-130a were measured by reverse-transcription quantitative PCR (RTqPCR) assay. Total RNA of LPS-induced C28/I2 cells was isolated using TRIzol reagent (Thermo Fisher Scientific, Waltham, MA, USA) according to the manufacturer's instructions. For the detection of IncRNA H19 or miR-130a expression, total RNA was reversely transcribed into cDNA using a High Capacity cDNA Reverse Transcription Kit (Thermo Fisher Scientific) or a microRNA Reverse Transcription Synthesis Kit (Thermo Fisher Scientific). Subsequently, qPCR analysis was performed using Maxima SYBR Green qPCR Master Mix $(2 \times)$ (Thermo Fisher Scientific) and specific primers. The relative expressions of H19 and miR-130a were calculated by $2^{-\Delta \Delta C t}$ method using GAPDH or U6 snRNA as an endogenous control for the normalization of H19 and miR-130a. The primers were as follows: H19 (Forward, 5'-GCAAGAAGCGGGTCTGTTT-3'; Reverse, 5'-GCTGGGTAGCACCATTTCTT-3'), GAPDH (Forward, 5'-TATGATGATATCAAGAGGGTAGT-3'; Reverse, 5'-TGTATC CAAACTCATTGTCATAC-3'). The primers for miR-130a and U6 were obtained from Ribobio (Guangzhou, China).

\section{Cell viability assay}

Cell viability was determined using Cell Counting Kit-8 (CCK8) (Dojindo, Tokyo, Japan) referring to the instructions of the manufacturer. In brief, C28/I2 cells during logarithmic growth period were seeded into 96-well microtiter plates at a density of $5 \times 10^{3}$ cells/well. Then, cells were transfected with indicated plasmids or oligonucleotides. After transfection for $48 \mathrm{~h}, 10 \mu \mathrm{L}$ of CCK-8 reagent was added to each well and incubated for another $4 \mathrm{~h}$ in a designated condition of $37^{\circ} \mathrm{C}, 5 \% \mathrm{CO}_{2}$. Absor- 
bance at $450 \mathrm{~nm}$ was measured to assess relative cell viability using a Microplate Reader (Bio-Rad, Hercules, CA, USA).

\section{Cell apoptosis assay}

Cell apoptosis was examined using an Annexin V-FITC Apoptosis Detection Kit (Abcam, Cambridge, UK) following the manufacturer's procedures. Generally, C28/I2 cells at a density of $1 \times 10^{5}$ cells/well were rinsed twice with cold PBS solution and resuspended in $1 \times$ Binding buffer, Afterwards, $5 \mu \mathrm{L}$ of Annexin V-FITC and $10 \mu \mathrm{L}$ of propidium iodide (PI) were introduced and incubated for $10 \mathrm{~min}$ in the dark. Finally, apoptotic cells were determined using a flow cytometer (BD Biosciences, San Jose, CA, USA).

\section{ELISA}

The levels of inflammatory cytokines interleukin-1 beta (IL$1 \beta$ ), interleukin-6 (IL-6), or tumor necrosis factor-alpha (TNF- $\alpha$ ) in culture supernatant were detected using Human IL-1 $\beta /$ IL-6/TNF- $\alpha$ Quantikine ELISA Kits (R\&D Systems, Abingdon, UK) according to the instructions supplied by the manufacturer.

\section{Luciferase reporter assay}

Bioinformatics analysis using Starbase online software (Sun Yat-sen University, Guangzhou, China) first proved the existence of putative binding sites between H19 and miR-130a. For luciferase assay, partial fragments of H19 containing the assumed miR-130a binding sites were amplified and sub-cloned into psiCHECK-2 vector (Promega, Madison, WI, USA) to generate wild-type H19 plasmid (H19-WT). Then, a Site-Directed Mutagenesis Kit (Thermo Fisher Scientific) was employed to generate mutated H19 plasmid (H19-MUT). H19-WT or H19MUT construct was co-transfected into C28/I2 cells along with miR-130a mimics or miR-NC. About $48 \mathrm{~h}$ post-transfection, relative luciferase activity was determined using the Dual-Luciferase reporter assay system (Promega).

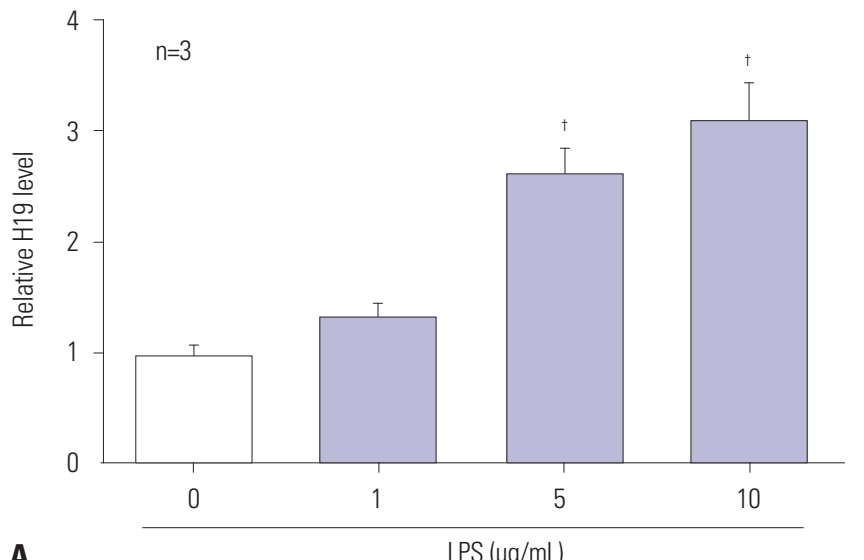

\section{RNA pull down}

RNA pull down was performed to detect the interplay between IncRNA H19 and miR-130a. In brief, C28/I2 cells were harvested and rinsed with cold PBS solution three times, followed by the addition of $1 \mathrm{~mL}$ of lysis buffer containing protease inhibitor (Solarbio, Beijing, China). Cell lysates were mixed with biotin-labeled miRNAs or lncRNA probe sequences, and incubated with M-280 streptavidincoated magnetic beads (Invitrogen) for $30 \mathrm{~min}$ at room temperature. Then, the beads were washed with ice-cold lysis buffer, low-salt buffer, and high-salt buffer, in this order, and bound RNAs was extracted using TRIzol reagent (Invitrogen) and quantified by RT-qPCR assay.

\section{Statistical analysis}

All experiments were performed three times. Statistical analysis was conducted using SPSS 20.0 (IBM Corp., Armonk, NY, USA), and results were presented as mean \pm standard deviation. Student's t-test or one-way analysis of variance was carried out to estimate significant group differences. $p<0.05$ represented a statistically significant result.

\section{RESULTS}

\section{H19 significantly upregulated in LPS-exposed C28/I2 cells}

A significant dose-dependent increase of $\mathrm{H} 19$ was observed in LPS-exposed C28/I2 cells (Fig. 1A). Next, C28/I2 cells treated with $5 \mu \mathrm{g} / \mathrm{mL}$ of LPS were transfected with si-H19 and its negative control (si-NC), respectively. As shown in Fig. 1B, the abundances of H19 were notably lessened in si-H19-transfected cells, compared to control, indicating that si-H19 could be used for the following loss-of-function trials.

Fig. 1. H19 is upregulated in LPS-treated C28/l2 cells in a dose-dependent manner. (A) C28//2 cells were treated with $0,1,5$, and $10 \mu \mathrm{g} / \mathrm{mL}$ of LPS for $12 \mathrm{~h}$, and then relative expression of $\mathrm{H} 19$ was measured by RT-qPCR. (B) LPS-exposed C28/l2 cells were transfected with si-NC or si-H19, followed by the detection of $\mathrm{H} 19$ expression by RT-qPCR. ${ }^{*} p<0.01,{ }^{\dagger} p<0.001$. LPS, lipopolysaccharide; RT-qPCR, reverse-transcription quantitative PCR. 


\section{H19 depletion rescues LPS-induced cell damage by modulating cell viability, apoptosis, and inflammation}

To explore the effect of H19 on LPS-induced cell injury, C28/ I2 cells were treated with LPS and transfected with si-NC or siH19. CCK-8 analysis showed that depletion of H19 promoted LPS-inhibited the viability of C28/I2 cells (Fig. 2A). Moreover, flow cytometry assay demonstrated that H19 knockdown attenuated the stimulatory effect of LPS on cell apoptosis (Fig. 2B). Consistently, ELISA assay indicated that inflammatory factors (IL-1 $\beta$, IL-6, TNF- $\alpha$ ) were markedly downregulated after the transfection of si-H19 in LPS-treated C28/I2 cells, compared with control (Fig. 2C-E). These findings suggested that H19 absence alleviated LPS-derived cell damage in OA.

\section{LncRNA H19, a molecular sponge for miR-130a}

Bioinformatics analysis revealed promising binding sites of miR-130a within H19 sequences, indicating a potential link between lncRNA H19 and miR-130a (Fig. 3A). Next, we further demonstrated the true interaction between the two by luciferase and RNA pull down assays. As presented in Fig. 3B and 3C, the luciferase activity of H19-WT observably declined in the presence of miR-130a, compared with miR-NC group in 293T cells; there was no significant alteration in H19-MUT reporter. Contrary to miR-130a, anti-miR-130a significantly increased the luciferase activity of H19-WT, while H19-MUT showed no distinct change. Next, biotin-labelled miR-130aWT (Bio-miR-130a-WT) or its mutant (Bio-miR-130a-MUT) devoid of specific H19 binding sites was constructed for RNA pull down analysis (Fig. 3D). Interestingly, we observed significant enrichment of IncRNA H19 in miR-130a-WT pulled down pellet as compared with negative control (Bio-NC), but less enrichment of H19 in miR-130a-MUT pulled down pellet (Fig. 3E). Likewise, wild-type biotin-labeled H19 probe (BioH19-probe-WT) could pull down miR-130a from RNA-protein complex, while Bio-H19-probe-MUT failed to enrich miR130a (Fig. 3F). Taken together, these data indicated that H19 was able to interplay with miR-130a through complementary sites in C28/I2 cells.

\section{Negative regulation between lncRNA H19 and miR-130a expression}

In present study, C28/I2 cells were transiently transfected with pcDNA-H19 or si-H19 to overexpress or suppress H19 (Fig. 4A). Then, miR-130a expression was measured by RT-qPCR assay. As presented in Fig. 4B, addition of $\mathrm{H} 19$ led to a loss of miR-130a expression, whereas H19 depletion induced miR130a expression, compared with their counterparts (Fig. 4B). Afterwards, miR-130a was overexpressed or interfered through transfection of miR-130a mimics or anti-miR-130a into C28/ I2 cells (Fig. 4C). Moreover, H19 was strikingly downregulated in miR-130a-transfected C28/I2 cells, but was remarkably increased in miR-130a-deficient C28/I2 cells (Fig. 4D). These findings indicated the negative relationship between lncRNA H19 and miR-130a expression.

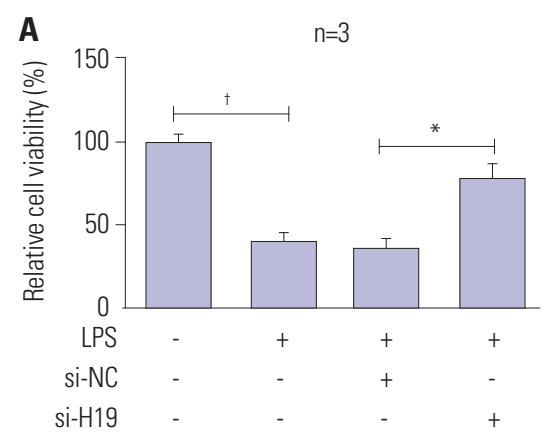

B

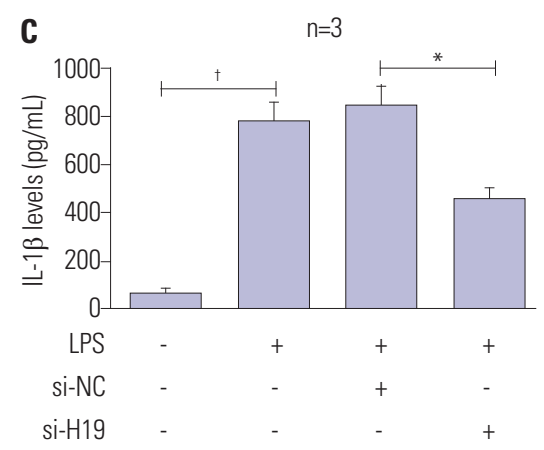

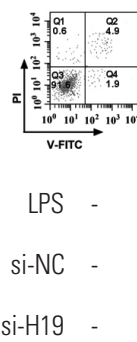

D

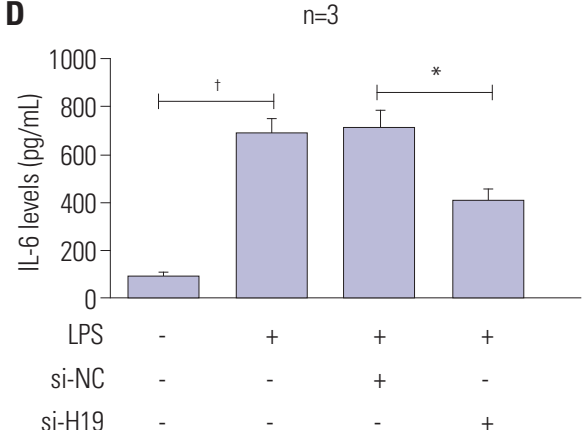

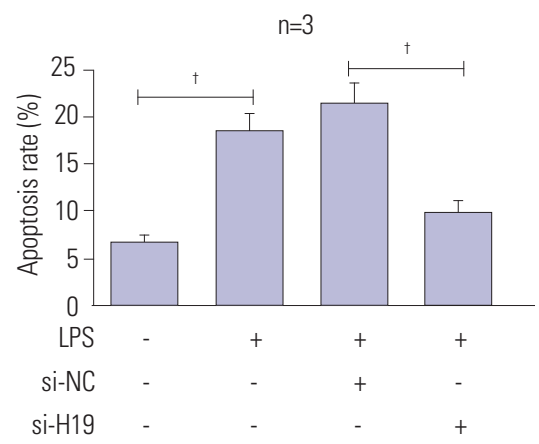

E

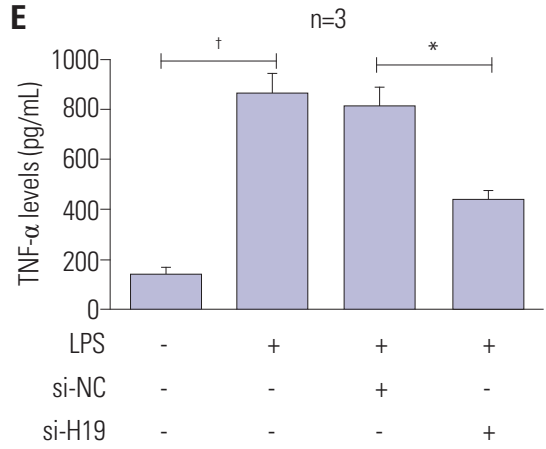

Fig. 2. LncRNA H19 deficiency remits LPS-induced cell damage in C28/I2 cells. After treatment with $5 \mu$ l of LPS, along with si-NC or si-H19, cell viability $(A)$, apoptosis (B), and the release of inflammatory cytokines (IL-1 $\beta$, IL-6, TNF- $\alpha$ ) (C-E) in C28/I2 cells were determined by CCK-8, flow cytometry, and ELISA assays. ${ }^{*} p<0.01,{ }^{\dagger} p<0.001$. LPS, lipopolysaccharide. 


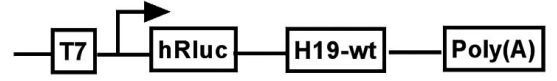

Target Location chr11:2016693-2016717[-]

H19-WT 5'-aauuugcacUAAGUCAUUUGCACUg-3' miR-130a $\quad\|\|_{\text {3'-uacgggaaAUU- -GU - AACGUGAC-5' }}$

H19-MUT 5'-aauuugcacGACGUAGUGCAACUGg-3'

A

Target Location chr11:2016693-2016717[-]

miR-130a 3'-uacgggaaaAUU - -GU- AACGUGAC-5' -WT

H19 5'-aauuugcacUAAGUCAUUUGCACUg-3'

miR-130a -MUT

D

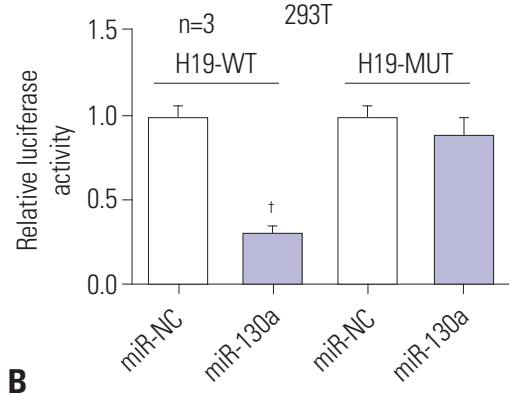

$\mathrm{C} 28 / \mathrm{I2}$

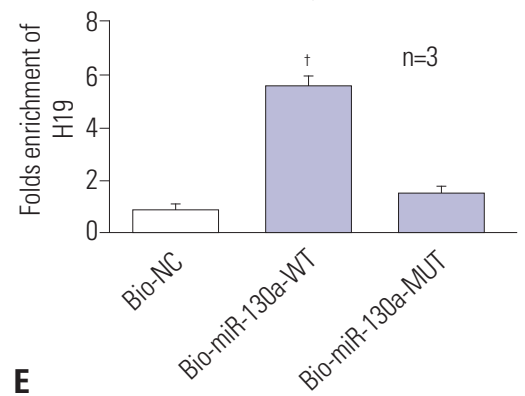

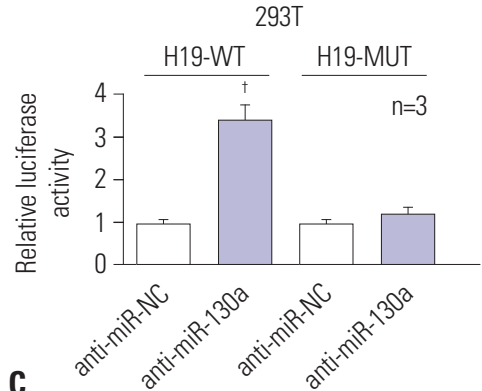

$\mathrm{C} 28 / 12$

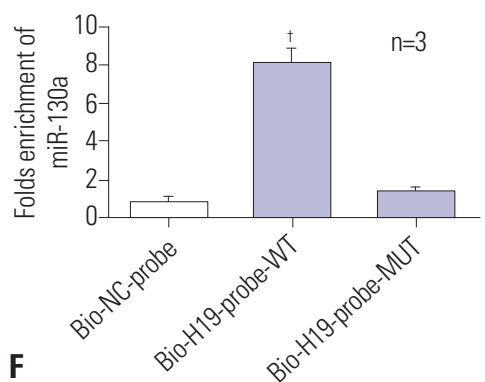

Fig. 3. LncRNA H19 identified as a molecular sponge for miR-130a. (A) Bioinformatics software was used to predict putative binding sites between miR130a and H19, as well as mutant sites in the H19-MUT reporter. (B and C) 293T cells were co-transfected with H19-WT or H19-MUT and miR-130a or anti-miR-130a for $48 \mathrm{~h}$, and then the activity of luciferase constructs focused on H19-WT and H19-MUT were determined by Dual-Luciferase reporter assay. (D) The mutant sites in the miR-130a-MUT reporter. (E and F) RNA pull down analysis was performed to verify the interplay between H19 and miR130a. ${ }^{\dagger} p<0.001$.
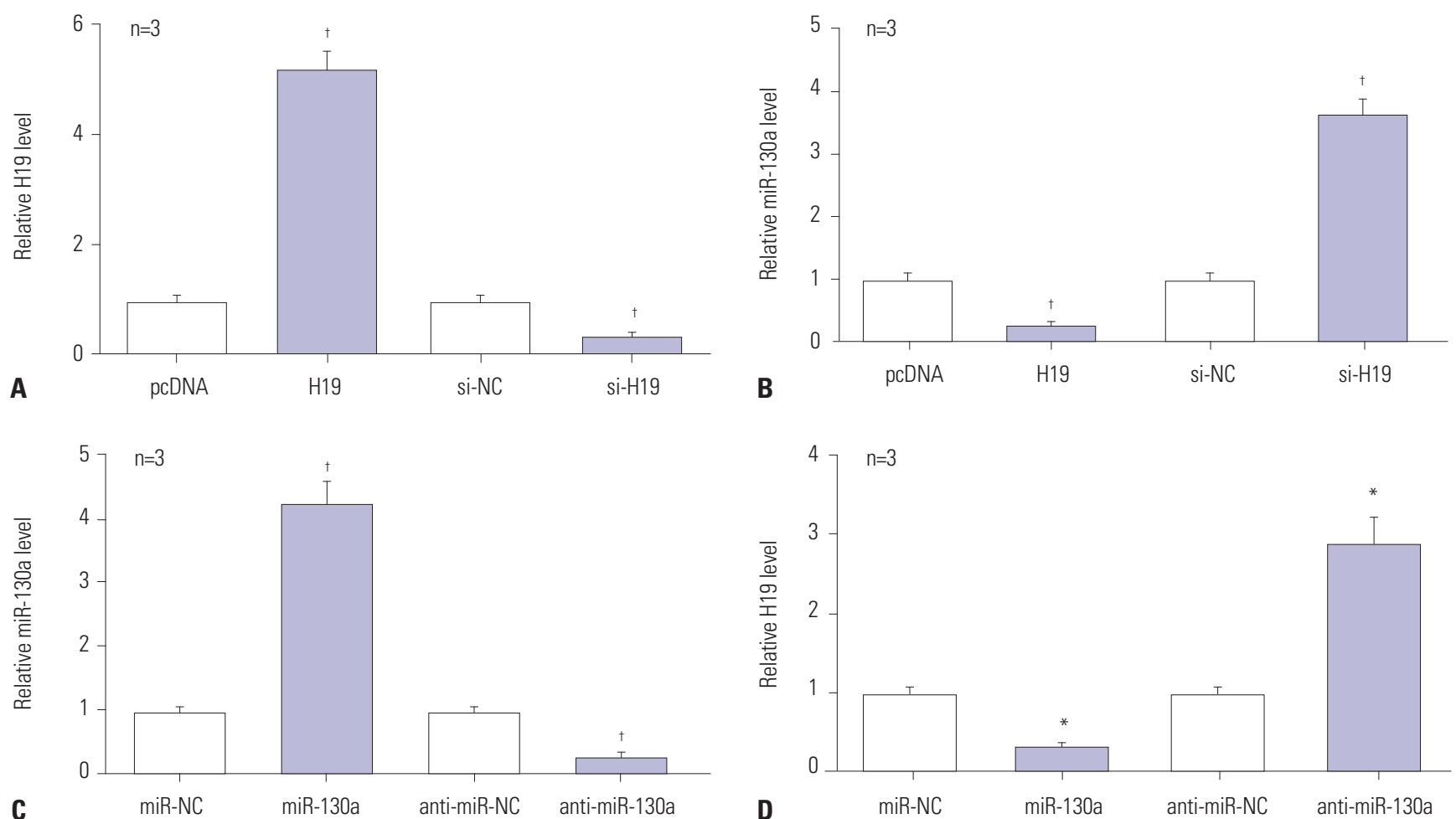

Fig. 4. The negative regulation between $\mathrm{H} 19$ and miR-130a in LPS-treated C28/l2 cells. (A) RT-qPCR assay was employed for the detection of $\mathrm{H} 19$ expression in $\mathrm{H} 19$ or si-H19-transfected cells. (B) The expression of miR-130a was negatively regulated by H19 in LPS-exposed C28/I2 cells. (C) RTqPCR assay was employed for the detection of miR-130a expression in miR-130a or anti-miR-130a-transfected cells. (D) The expression of H19 was negatively regulated by miR-130a in LPS-exposed C28/I2 cells. ${ }^{*} p<0.01,{ }^{\dagger} p<0.001$. LPS, lipopolysaccharide; RT-qPCR, reverse-transcription quantitative PCR. 
Effects of H19 on cell viability, apoptosis, and inflammation abrogated by miR-130a restoration miR-130a expression in LPS-damaged C28/I2 cells was first detected by RT-qPCR, and the results revealed marked downregulation of miR-130a in a dose-dependent manner (Fig. $5 \mathrm{~A})$. Further, restoration experiments were performed to investigate the impacts of miR-130a on H19-mediated cell injury through transfection of pcDNA3.1, H19, H19+miR-NC, or H19+miR-130a into LPS-treated C28/I2 cells. As demonstrated in Fig. 5B and C, restoration of miR-130a abolished the inhibitory effect of $\mathrm{H} 19$ on cell viability. Moreover, the alteration of cell apoptosis, induced by H19, was also reversed by miR130a introduction. In terms of inflammatory cytokines, the levels of IL- $1 \beta$, IL- 6 , and TNF- $\alpha$ were notably decreased in H19+miR-130a transfected cells, compared with its control (Fig. 5D-F), hinting that miR-130a weakened H19-stimulated inflammation in LPS-treated C28/I2 cells. Our findings indicated that overexpression of miR-130a abrogated the damaging effects of H19 on LPS-exposed C28/I2 cells.

\section{DISCUSSION}

This report provides the first evidence that lncRNA H19 silencing significantly rescue LPS-induced cell injury by promoting cell viability and suppressing apoptosis and inflammatory cy- tokines release in C28/I2 cells. Overall, we found H19 to be a molecular sponge for miR-130a. Exogenous overexpression of miR-130a ameliorated H19-induced cell injury in LPS-treated C28/I2 cells.

LPS is generally known as a major mediator in bacterial infection and disease evolution, and plays a key role in the induction of strong inflammatory responses, including production of proinflammatory cytokines and chemokines. As stated by Lee, et al., ${ }^{20}$ LPS contributes to the production of cell adhesion molecules, TNF- $\alpha$, and activated NF- $\kappa \mathrm{B}$, and induces the adhesion/migration of monocytes to human umbilical vein endothelial cell. Li, et al. ${ }^{21}$ reported that LPS triggers cell proliferation, suppresses apoptosis, and enhances the release of IL- $1 \beta$, IL-6, IL-12, and TNF- $\alpha$ in gastric cancer. Our study showed that LPS significantly induces chondrocyte injury, reflected by decreased cell viability, increased apoptosis, and stimulation of IL- $1 \beta$, IL- 6 , and TNF- $\alpha$ secretion in C28/I2 cells, which was similar with previous research reported by Sun, et al. ${ }^{22}$

Increasing evidence has demonstrated the biological functions of lncRNA in various diseases by the modulation of $\mathrm{X}$ chromosome silencing, genomic imprinting, chromatin modification, transcription, and translation. ${ }^{23,24}$ In OA, a series of lncRNAs with abnormal expression plays pivotal roles in the occurrence and development of this disease. For instance, an abundance of lncRNA RP11-445H22.4 in LPS-induced cartilage ATDC5 cells has been found to result in a decrease in cell
A

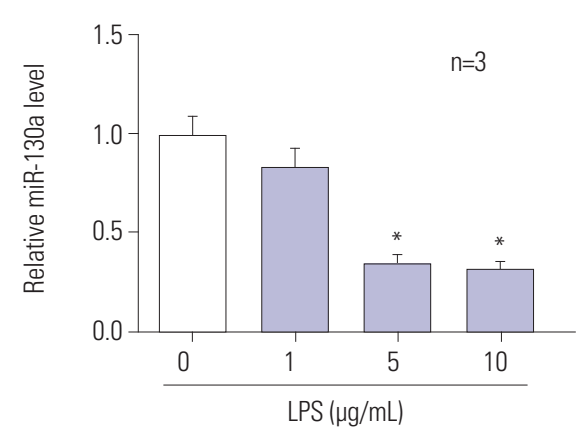

D

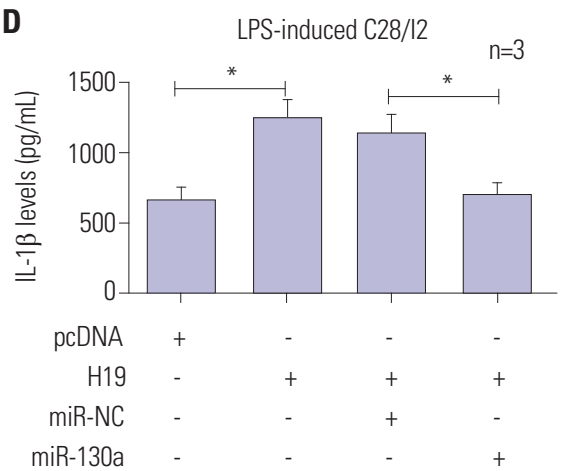

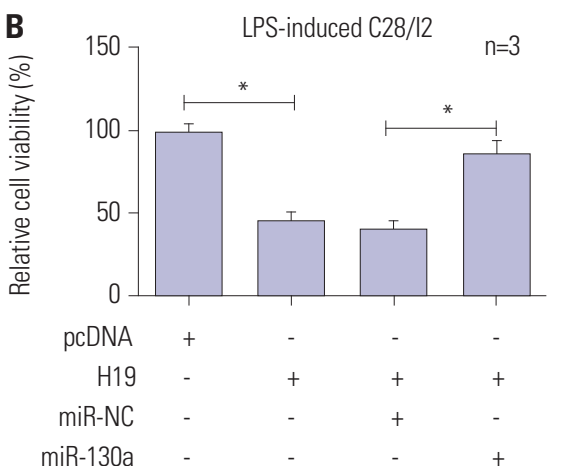

E

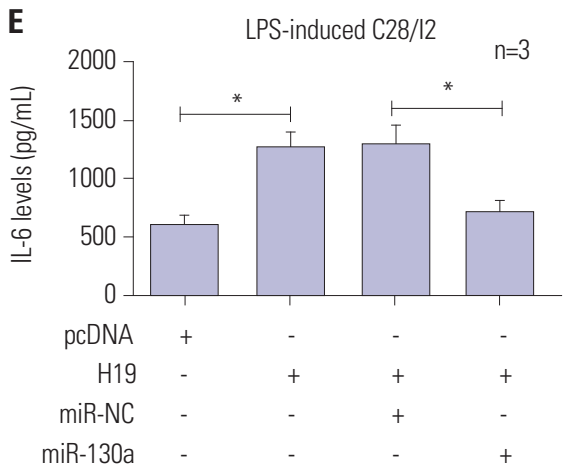

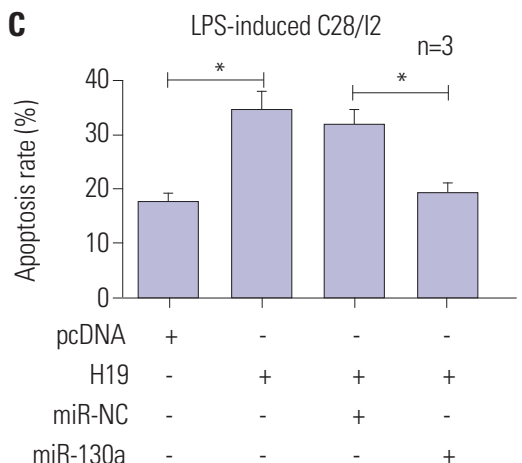

$\mathbf{F}$

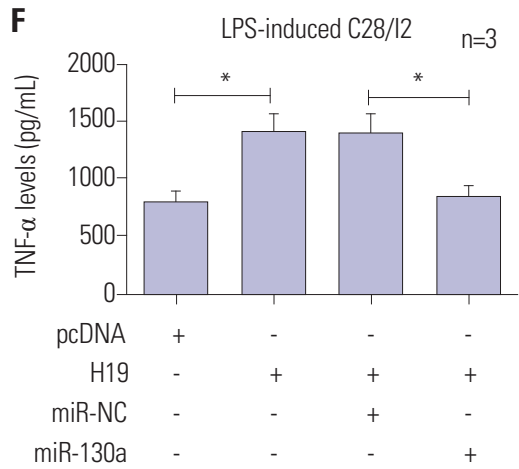

Fig. 5. Effects of $\mathrm{H} 19$ are abrogated by miR-130a overexpression. (A) C28/l2 cells were treated with $0,1,5$, and $10 \mu \mathrm{g} / \mathrm{mL}$ of LPS for $12 \mathrm{~h}$, and then relative expression of miR-130a was determined by RT-qPCR. LPS-induced C28/l2 cells were transfected with pcDNA3.1, H19, H19+miR-NC, or H19+miR130a. At about $48 \mathrm{~h}$ post-transfection, cell viability (B), apoptosis (C), and the levels of inflammatory cytokines (IL-1 $\beta$, IL-6, TNF- $\alpha$ ) (D-F) were measured by CCK-8, flow cytometry, and ELISA assays. * $p<0.01$. LPS, lipopolysaccharide; RT-qPCR, reverse-transcription quantitative PCR. 
viability, increase in apoptosis, and secretion of inflammatory cytokines. $^{22}$ Ectopic expression of PVT1 was shown to be involved in the modulation of chondrocyte apoptosis, which further led to the degeneration of cartilage. ${ }^{25}$ In contrast to previous studies, our data indicated that H19 is downregulated in LPS-damaged C28/I2 cells. Functionally, knockdown of H19 relieved LPS-induced chondrocyte injury, as evidenced by increased cell viability, increased apoptosis, and reduced inflammatory cytokines releases. Therefore, our study demonstrated the protective role of $\mathrm{H} 19$ in OA chondrocytes, which was consistent with a previous study. ${ }^{17}$

LncRNAs are considered to be a major players of competing endogenous RNA (ceRNA)-mediated network due to its regulatory effect on target gene expression by serving as a molecular sponge for specific miRNA. ${ }^{26}$ Ectopic expressed lncRNA is associated with the occurrence and development of a range of diseases, including $\mathrm{OA}$. As reported by $\mathrm{Xu}$ and $\mathrm{Xu},{ }^{27}$ downregulated lncRNA MEG3 induces cell proliferation but inhibits apoptosis in IL-1 $\beta$-derived rat chondrocytes via a miR-1/ SMAD7 axis. Li, et al. ${ }^{28}$ suggested that lncRNA CIR upregulates MMP13 expression by competitively sponging miR-27b and then induces the degradation of chondrocyte extracellular matrix in OA. Zhang, et al. ${ }^{29}$ revealed that lncRNA UFCl regulates the proliferation and apoptosis of OA chondrocytes through physical interaction with miR-34a, hinting at the pivotal role of UFC1 in OA remission. We confirmed that H19 negatively regulates miR-130a in chondrocytes through different methods, including luciferase activity, RNA pull down, and RT-qPCR. Furthermore, miR-130a was downregulated in LPSstimulated chondrocytes.

MiR-130a is implicated in the regulation of a broad range of cell phenotypes. For instance, upregulation of miR-130a was found to promote the migration and invasion in breast cancer cells through directly targeting Dicer mRNA. ${ }^{30}$ Targeting of RUNX3 by miR-130a was shown to contribute to cell proliferation in gastric cancer. ${ }^{31}$ In inflammatory disease, miR-130a lowered the expression levels of inflammatory cytokines in patients with acute ischemic stroke. ${ }^{32}$ Also, miR-130a alleviated inflammatory responses and human coronary artery endothelial cell injury via regulation of PTEN/PI3K/Akt/eNOS pathway. ${ }^{33}$ In line with previous studies, we showed that overexpression of miR-675 overturned H19-inhibited cell viability and induced apoptosis and inflammatory cytokines in LPS-treated C28/I2 cells, implying that $\mathrm{H} 19$ aggravates LPS-induced C28/I2 cell damage by interacting with miR-130a. Although the molecular mechanism of the H19/miR-130a axis in chondrocytes has been illustrated in vitro, there is also need to explore the signaling pathway mediated by H19/miR-130a axis and the modulation of H19 in OA treatments.

Although primary chondrocytes isolated from animal and human sources have served as available models for studying the mechanisms of cell growth and inflammation, ${ }^{34}$ research has also confirmed the limitations of using primary chondro- cytes. As stated by Goldring, ${ }^{35}$ due to the uncontrollability of cartilage sources, sufficient numbers of chondrocytes cannot be readily obtained from random operative procedures. Moreover, the phenotypic stability and proliferative capacity in adult human chondrocytes is quickly lost after continuous passage culture. Finger, et al. ${ }^{36}$ also reported that primary human adult articular chondrocytes are difficult to obtain and show little proliferative activity. Compared to primary chondrocytes, immortalized C28/I2 cell line can be cultured steadily and has the ability to proliferate rapidly in designated medium. ${ }^{37}$ Thus, we selected LPS-induced C28/I2 cell lines as a disease model to investigate proliferation, apoptosis, and inflammation in OA. However, C28/I2 cells may exhibit less correlation with a true disease model, compared with primary chondrocytes, and thus, further exploration of the role of the H19/miR-130a axis in primary chondrocytes is needed in subsequent research.

In summary, our data revealed that lncRNA H19 suppresses viability, induces apoptosis, and increases inflammatory cytokines release in LPS-derived chondrocytes by acting as a molecular sponge for miR-130a. These findings highlight a novel pathological mechanism of OA, hinting at a potential biological target for OA treatment.

\section{AUTHOR CONTRIBUTIONS}

Conceptualization: Yi Hu. Data curation: Sukai Li. Formal analysis: Yi Hu. Investigation: Yi Hu, Sukai Li. Methodology: Sukai Li. Project administration: Yi Hu. Resources: Yi Hu. Software: Yonggen Zou. Validation: Sukai Li. Writing-original draft: Yonggen Zou. Writing-review \& editing: Yonggen Zou.

\section{ORCID iDs}

Yi Hu

Sukai Li

Yonggen Zou

https://orcid.org/0000-0002-9040-7394

https://orcid.org/0000-0002-4246-1038

https://orcid.org/0000-0002-1281-308X

\section{REFERENCES}

1. Poulet B, Staines KA. New developments in osteoarthritis and cartilage biology. Curr Opin Pharmacol 2016;28:8-13.

2. Wu C, Tian B, Qu X, Liu F, Tang T, Qin A, et al. MicroRNAs play a role in chondrogenesis and osteoarthritis (review). Int J Mol Med 2014;34:13-23.

3. Findlay DM, Atkins GJ. Osteoblast-chondrocyte interactions in osteoarthritis. Curr Osteoporos Rep 2014;12:127-34.

4. Cross M, Smith E, Hoy D, Nolte S, Ackerman I, Fransen M, et al. The global burden of hip and knee osteoarthritis: estimates from the global burden of disease 2010 study. Ann Rheum Dis 2014;73:132330.

5. Chen WK, Yu XH, Yang W, Wang C, He WS, Yan YG, et al. lncRNAs: novel players in intervertebral disc degeneration and osteoarthritis. Cell Prolif 2017;50:e12313.

6. World report on disability. Eur J Disabil Res 2011;5:136.

7. Musumeci G, Castrogiovanni P, Trovato FM, Weinberg AM, Al-Wasiyah MK, Alqahtani MH, et al. Biomarkers of chondrocyte apopto- 
sis and autophagy in osteoarthritis. Int J Mol Sci 2015;16:20560-75.

8. Hwang HS, Kim HA. Chondrocyte apoptosis in the pathogenesis of osteoarthritis. Int J Mol Sci 2015;16:26035-54.

9. Zamli Z, Adams MA, Tarlton JF, Sharif M. Increased chondrocyte apoptosis is associated with progression of osteoarthritis in spontaneous Guinea pig models of the disease. Int J Mol Sci 2013;14: 17729-43.

10. Schuerwegh AJ, Dombrecht EJ, Stevens WJ, Van Offel JF, Bridts CH, De Clerck LS. Influence of pro-inflammatory (IL-1 alpha, IL-6, TNF-alpha, IFN-gamma) and anti-inflammatory (IL-4) cytokines on chondrocyte function. Osteoarthritis Cartilage 2003;11:681-7.

11. Huang ZY, Stabler T, Pei FX, Kraus VB. Both systemic and local lipopolysaccharide (LPS) burden is associated with knee osteoarthritis (OA). Osteoarthritis Cartilage 2016;24(Suppl 1):S329-30.

12. Zhao C, Wang Y, Jin H, Yu T. Knockdown of microRNA-203 alleviates LPS-induced injury by targeting MCL-1 in C28/I2 chondrocytes. Exp Cell Res 2017;359:171-8.

13. Marques AC, Ponting CP. Intergenic lncRNAs and the evolution of gene expression. Curr Opin Genet Dev 2014;27:48-53.

14. Kataoka M, Wang DZ. Non-coding RNAs including miRNAs and lncRNAs in cardiovascular biology and disease. Cells 2014;3:88398.

15. Liu Q, Hu X, Zhang X, Dai L, Duan X, Zhou C, et al. The TMSB4 pseudogene lncRNA functions as a competing endogenous RNA to promote cartilage degradation in human osteoarthritis. Mol Ther 2016;24:1726-33.

16. Han Y, Ma J, Wang J, Wang L. Silencing of H19 inhibits the adipogenesis and inflammation response in ox-LDL-treated Raw264.7 cells by up-regulating miR-130b. Mol Immunol 2018;93:107-14.

17. Steck E, Boeuf S, Gabler J, Werth N, Schnatzer P, Diederichs S, et al. Regulation of $\mathrm{H} 19$ and its encoded microRNA-675 in osteoarthritis and under anabolic and catabolic in vitro conditions. J Mol Med (Berl) 2012;90:1185-95.

18. Zheng H, Dong X, Liu N, Xia W, Zhou L, Chen X, et al. Regulation and mechanism of mouse miR-130a/b in metabolism-related inflammation. Int J Biochem Cell Biol 2016;74:72-83.

19. Li ZC, Han N, Li X, Li G, Liu YZ, Sun GX, et al. Decreased expression of microRNA-130a correlates with TNF- $\alpha$ in the development of osteoarthritis. Int J Clin Exp Pathol 2015;8:2555-64.

20. Lee W, Yang EJ, Ku SK, Song KS, Bae JS. Anti-inflammatory effects of oleanolic acid on LPS-induced inflammation in vitro and in vivo. Inflammation 2013;36:94-102.

21. Li K, Dan Z, Nie Y, Hu X, Gesang L, Bianba Z, et al. CD14 knockdown reduces lipopolysaccharide-induced cell viability and expression of inflammation-associated genes in gastric cancer cells in vitro and in nude mouse xenografts. Mol Med Rep 2015;12:4332-9.

22. Sun T, Yu J, Han L, Tian S, Xu B, Gong X, et al. Knockdown of long non-coding RNA RP11-445H22.4 alleviates LPS-induced injuries by regulation of MiR-301a in osteoarthritis. Cell Physiol Biochem 2018;45:832-43.

23. Khorkova O, Hsiao J, Wahlestedt C. Basic biology and therapeutic implications of lncRNA. Adv Drug Deliv Rev 2015;87:15-24.

24. Quan M, Chen J, Zhang D. Exploring the secrets of long noncoding RNAs. Int J Mol Sci 2015;16:5467-96.

25. Li Y, Li S, Luo Y, Liu Y, Yu N. LncRNA PVT1 regulates chondrocyte apoptosis in osteoarthritis by acting as a sponge for miR-488-3p. DNA Cell Biol 2017;36:571-80.

26. Tay Y, Karreth FA, Pandolfi PP. Aberrant ceRNA activity drives lung cancer. Cell Res 2014;24:259-60.

27. $\mathrm{Xu}$ J, Xu Y. The lncRNA MEG3 downregulation leads to osteoarthritis progression via miR-16/SMAD7 axis. Cell Biosci 2017;7:69.

28. Li YF, Li SH, Liu Y, Luo YT. Long noncoding RNA CIR promotes chondrocyte extracellular matrix degradation in osteoarthritis by acting as a sponge for Mir-27b. Cell Physiol Biochem 2017;43:60210.

29. Zhang G, Wu Y, Xu D, Yan X. Long noncoding RNA UFC1 promotes proliferation of chondrocyte in osteoarthritis by acting as a sponge for miR-34a. DNA Cell Biol 2016;35:691-5.

30. He L, Wang HY, Zhang L, Huang L, Li JD, Xiong Y, et al. Prognostic significance of low DICER expression regulated by miR-130a in cervical cancer. Cell Death Dis 2014;5:e1205.

31. Lee SH, Jung YD, Choi YS, Lee YM. Targeting of RUNX3 by miR130a and miR-495 cooperatively increases cell proliferation and tumor angiogenesis in gastric cancer cells. Oncotarget 2015;6: 33269-78.

32. Jin F, Xing J. Circulating miR-126 and miR-130a levels correlate with lower disease risk, disease severity, and reduced inflammatory cytokine levels in acute ischemic stroke patients. Neurol Sci 2018;39: 1757-65.

33. Song CL, Liu B, Shi YF, Liu N, Yan YY, Zhang JC, et al. MicroRNA130a alleviates human coronary artery endothelial cell injury and inflammatory responses by targeting PTEN via activating PI3K/ Akt/eNOS signaling pathway. Oncotarget 2016;7:71922-36.

34. Goldring MB. The role of the chondrocyte in osteoarthritis. Arthritis Rheum 2000;43:1916-26.

35. Goldring MB. Immortalization of human articular chondrocytes for generation of stable, differentiated cell lines. Methods Mol Med 2004;100:23-36.

36. Finger F, Schörle C, Zien A, Gebhard P, Goldring MB, Aigner T. Molecular phenotyping of human chondrocyte cell lines T/C-28a2, T/ C-28a4, and C-28/I2. Arthritis Rheum 2003;48:3395-403.

37. Loeser RF, Sadiev S, Tan L, Goldring MB. Integrin expression by primary and immortalized human chondrocytes: evidence of a differential role for $\alpha 1 \beta 1$ and $\alpha 2 \beta 1$ integrins in mediating chondrocyte adhesion to types II and VI collagen. Osteoarthritis Cartilage 2000;8:96-105. 